\title{
Analysis of workpiece geometry change during isothermal free deformation of titanium alloys in square die
}

\author{
Larin Sergey Nikolaevich \\ Head of department of "Mechanics of Plastic Forming" \\ Tula State University, \\ Tula, Russia
}

\author{
Platonov Valeriy Ivanovich \\ Assistant professor of department of \\ "Mechanics of Plastic Forming" \\ Tula State University, \\ Tula, Russia
}

\author{
Bessmertnaya Yuliya Vyacheslavovna \\ Assistant professor of department of \\ "Mechanics of Plastic Forming" \\ Tula State University, \\ Tula, Russia
}

\begin{abstract}
Multi-layer cellular workpieces are used to manufacture fuel tanks, as well as the external hulls of aircraft. These designs are characterized by low weight and high strength. Monotonic formation with inert gases looks promising for the manufacturing of large workpieces. This article presents an approach to the modeling of energy-force parameters during free forming of titanium alloy workpieces in square dies with shortterm creep. The results of mathematical modeling of the investigated process are given in the form of inequations which allow estimating pressure and critical modes. According to these inequations, we found the influence of the process time on blank's relative thickness change in various points of its surface, as well as on its height and maximum damage rate change.
\end{abstract}

Keywords- pneumatic forming, deformations, square die, height, thickness.

\section{INTRODUCTION}

Structures consisting of one or several cellular sheet layers are used to manufacture fuel tanks, as well as the external hulls of parts, wings, fairings, etc [1-4]. These structures are characterized by increased strength and at the same time they have low weight and high resistance to various loading levels. The cellular structures are manufactured using mainly the mechanical and electrochemical processing technologies [5-9]. Concerning the manufacturing of these structures, slow deformation at high temperatures with excess gas pressure is one of the most promising technologies.

\section{RESULTS AND DISCUSSION}

This paper presents an approach to the creation of a model, describing the energy-force parameters for the free forming of titanium alloy workpieces in square dies with short-term creep. Therefore let us consider the forming of a sheet material with thickness $h_{0}$ in a square die with sides equaling $2 a$ during the short-term creep mode under pressure $p$, which varies according to time $t$ in the following form: $p=p_{0}+a_{p} t^{n} p$, where $a_{p}$ and $n_{p}$ are the loading rule parameters. The material exhibits anisotropic properties. The forming blank was obtained from the sheet material with all sides parallel to the axis $y$ (perpendicular to the rolling direction) [1-3]. The workpiece itself was fixed along the outer contour (figure).

In modeling, we assume that the dimensions of the workpiece are such that there is a plane stress state $\left(\sigma_{z}=0\right)$. During the forming, the workpiece obtains a spherical crosssection. In case of deformation, we assume that the crosssection is a circle along the symmetry axes.

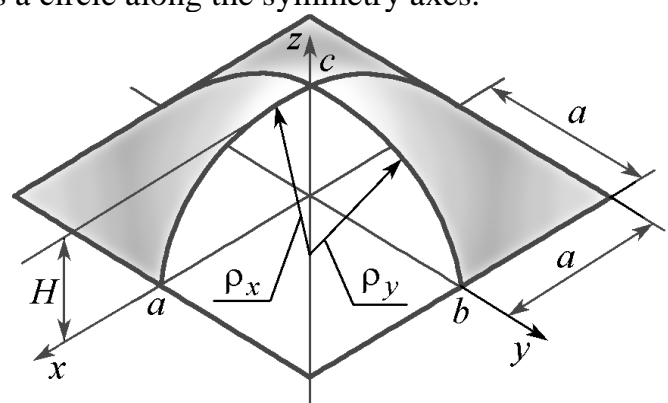

Fig. 1. Scheme for modeling the square workpiece forming

Now we need to find the value of the damage rate $\omega_{e c}^{c}$ at the pole of the layer, accumulated during the forming. For this purpose, it is required to substitute the value of $\sigma_{e}$ from the first equation of state into the second. Then we get

$$
\dot{\omega}_{e c}^{c}=\frac{k}{B} \xi_{e}^{c} ; \quad k=B / \varepsilon_{e n p}^{c} .
$$


The next step is to find the workpiece loading, when $\xi_{e c}^{c}=\xi_{e c 1}^{c}=$ const at its pole. In this case, having integrated the equations (1) under the initial conditions $t=0, \omega_{e c}^{c}=0$, we get:

$$
\omega_{e c}^{c}=\frac{k}{B} \xi_{e c 1}^{c} t=\frac{k \varepsilon_{e c}^{c}}{B} .
$$

Critical deformations in the workpiece occur when $\omega_{e c}^{c}=1$, whence it follows that:

$$
\varepsilon_{\text {ecnp }}^{c}=\frac{B}{k} .
$$

Pressure $p$, which creates conditions for the successful forming, can be found according to the following function:

$$
p=\frac{2 \sigma_{e 0}\left(1-\omega_{e c}^{c}\right)^{m / n} H h_{0} a^{2}\left(1+\chi_{1}\right)}{D_{1}\left(H^{2}+a^{2}\right)^{3}}\left(\frac{\xi_{e c 1}^{c}}{B}\right)^{1 / n} .
$$

Correspondence $\omega_{e c}^{c}=\omega_{e}^{c}(t)$ is determined by the relation (1), and the function $H=H(t)$ can be found according to the following equation:

$$
\varepsilon_{e c}^{c}=\int_{0}^{t} \xi_{e c}^{c} d t=\xi_{e c 1}^{c} t
$$

and as follows:

$$
\varepsilon_{e c}^{c}=\int_{0}^{t} C_{1} \xi_{y c}^{c} d t=\int_{0}^{H} C_{1} \frac{2 H d H}{H^{2}+a^{2}}
$$

that is:

$$
t=\frac{C_{1}}{\xi_{e c 1}^{c}} \ln \frac{a^{2}}{H^{2}+a^{2}} .
$$

Let us consider the loading of a sheet blank when $p=$ const .

Substituting

$$
\xi_{e c}^{c}=C_{1} \xi_{y c}^{c}=C_{1} \frac{2 H \dot{H}}{H^{2}+a^{2}}
$$

$\xi_{x c}^{c}=\xi_{y c}^{c}=\frac{2 H \dot{H}}{H^{2}+a^{2}} ; \xi_{z c}^{c}=\frac{\dot{h}}{h}$, into the equation (1) and taking onto account (3) we obtain:

$$
\dot{\omega}_{e c}^{c}=\frac{k}{B} C_{1} \frac{2 H \dot{H}}{H^{2}+a^{2}}=\frac{2 C_{1} H \dot{H}}{\left(H^{2}+a^{2}\right) \varepsilon_{e c n p}^{c}} .
$$

Having integrated this equation under the initial conditions $t=0, \omega_{e c}^{c}=0$, we have:

$$
\omega_{e c}=\frac{1}{\xi_{e c 1}^{c}} \ln \frac{a^{2}}{H^{2}+a^{2}} .
$$

The maximum height at the pole of the workpiece $H_{*}$ can be found from (9) with $\omega_{e c}^{c}=1$.

Let us consider the stress and deformation states in the point $x=a, y=0$.
The values of $\sigma_{e a}$ and $\xi_{e a}^{c}$ for this point are determined according to the relations $\sigma_{e a}=\bar{D}_{1 a} \sigma_{x a}$, and $\xi_{e a}^{c}=\bar{C}_{1 a} \xi_{x a}^{c}$. The damage rate is found from equation

$$
\dot{\omega}_{e a}^{c}=\frac{\xi_{e a}^{c}}{\varepsilon_{e a n p}} .
$$

In case of sheet blank loading, when $\xi_{e a}^{c}=\xi_{e a 1}^{c}=$ const,

$$
\omega_{e a}^{c}=\frac{\xi_{e a 1}^{c} t}{\varepsilon_{e a n p}} .
$$

Critical deformations in the workpiece are reached at $\omega_{e a}^{c}=1$.

The pressure, at which the conditions for the final forming are realized, is obtained by substituting $\sigma_{e a}$ и $\xi_{e a}^{c}=\xi_{e a 1}^{c}$ into the first equation. Then we get:

$$
p(t)=\frac{2 \sigma_{e 0}\left(1-\omega_{e a}^{c}\right)^{m / n} h_{0} a^{2} H}{\left(H^{2}+a^{2}\right)^{2}}\left(\frac{\xi_{e a 1}^{c}}{B}\right)^{1 / n} .
$$

Function $H=H(t)$ is found from:

$$
\varepsilon_{e a}^{c}=\int \xi_{e a 1}^{c} d t=\xi_{e a 1}^{c} t=\int \bar{C}_{1 a} \xi_{x a}^{c} d t
$$

Thus,

$$
t=\frac{\bar{C}_{1 a}}{\xi_{e a 1}^{c}} \int_{0}^{H} \frac{2 H d H}{H^{2}+a^{2}}=\frac{\bar{C}_{1 a}}{\xi_{e a 1}^{c}} \ln \frac{H^{2}+a^{2}}{a^{2}} .
$$

If the loading is carried out under the condition that $p=$ const , the accumulation of damage rate is described by equation:

$$
\dot{\omega}_{e a}=\frac{\xi_{e a}^{c}}{\varepsilon_{e a n p}^{c}}=\frac{\bar{C}_{1 a} \xi_{x a}^{c}}{\varepsilon_{e a n p}^{c}}=\frac{\bar{C}_{1 a} 2 H \dot{H}}{\varepsilon_{e a n p}^{c}\left(H^{2}+a^{2}\right)} .
$$

Integration of this equation under the initial conditions $t=0, \omega_{e a}^{c}=0$ leads to the following relation:

$$
\omega_{e a}^{c}=\frac{\bar{C}_{1 a}}{\varepsilon_{e a n p}^{c}} \ln \frac{H^{2}+a^{2}}{a^{2}} .
$$

The value of $H_{*}$ is calculated under the condition that $\omega_{e a}^{c}=1$. The non-dimensional destruction time $\bar{t}_{*}$ is determined by the equation:

$$
\bar{t}_{*}=\int_{0}^{H *} \frac{\left(1-\omega_{e a}^{c}\right)^{m} H^{n+1} a^{2 n} h_{0}^{n} d H}{\left(H^{2}+a^{2}\right)^{2 n+1}},
$$

where

$$
\bar{t}_{*}=\frac{p^{n} \bar{D}_{1 a}^{n} B}{\bar{C}_{1 a} \sigma_{e a}^{n} 2^{n+1}} t_{*} .
$$

Deformation conditions in the point $x=0, y=a$ are similar to these conditions in the point $y=0, x=a$.

The obtained solution for finding the energy-force parameters during the isothermal forming of a flat workpiece made of anisotropic material can be considered as valid for the 
workpiece made of a material without anisotropic properties. Therefore we assume that $R_{x}=R_{y}=1$.

\section{CONCLUSION}

Using the obtained equations, we can estimate the feasibility of titanium alloy forming, obeying the kinetic theory of creep and damage. The evaluation of the obtained correspondences (figures 2 and 3) shows that the growth of the loading parameters $a_{p}$ and $n_{p}$ can lead to a noticeable increase in the height of the forming workpiece $\bar{H}$ and a decrease in the thickness of the hull in the upper part of the workpiece $\bar{h}_{c}$ with deformation time $t$. The critical height $\bar{H}_{*}$ and thickness $\bar{h}_{c^{*}}$ of these values do not change.

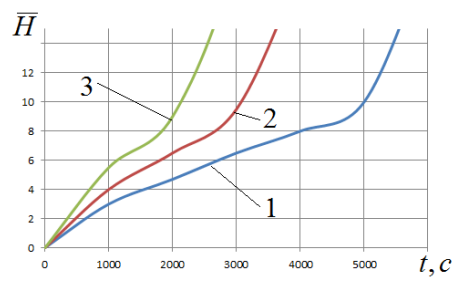

a)

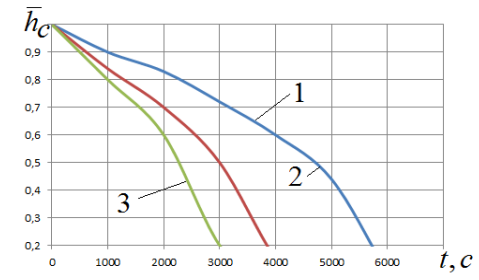

b)

Fig. 2. Evaluation of forming time influence on $\bar{H}$ (a) and $\bar{h}_{c}$ (b) for a titanium alloy $6 \mathrm{Al}-4 \mathrm{~V}\left(T=930^{\circ} \mathrm{C} ; n_{p}=0.4 ; a=15 \mathrm{~mm}\right): 1$ $a_{p}=0.04 \mathrm{MPa} / \mathrm{s}^{n}{ }^{p} ;-a_{p}=0.06 \mathrm{MPa} / \mathrm{s}^{{ }^{n} p} ; 3-a_{p}=0.08 \mathrm{MPa} / \mathrm{s}^{n_{p}}$

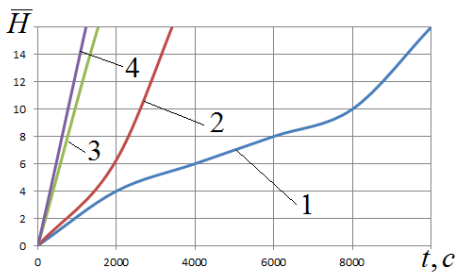

a)

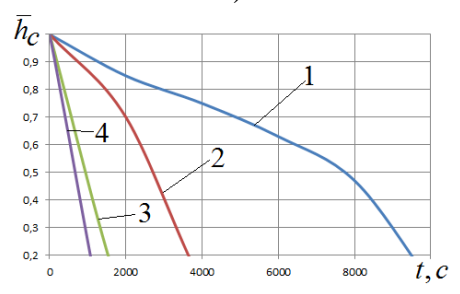

b)

Fig. 3. Evaluation of forming time influence on $\bar{H}$ (a) and $\bar{h}_{c}$ (b) for a titanium alloy $6 \mathrm{Al}-4 \mathrm{~V}\left(T=930^{\circ} \mathrm{C} ; a_{p}=0.06 \mathrm{MPa} / \mathrm{s}^{n} p ; a=15 \mathrm{~mm}\right)$ : $1-n_{p}=0.3 ; 2-n_{p}=0.4 ; 3-n_{p}=0.5 ; 4-n_{p}=0.6$
Fig. 4 shows the correspondence, which helps to reveal the forming time influence on the material damage rate. It shows that the critical value of the accumulated damage $\omega_{e}$ is observed at the workpiece pole and that with the increase of forming time, its value also increases (fig. 4).

It can be noted that the loading conditions do not affect the damage rate under conditions of viscous metal flow. There is also a strong influence of the loading parameters $a_{p}$ and $n_{p}$ on time when destruction $t_{*}$ is possible.

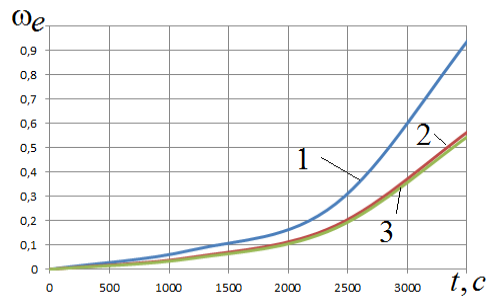

Fig. 4. Dependence of $\omega_{e}$ from $t$ for the titanium alloy 6Al-4V $\left(T=930^{\circ} \mathrm{C}\right.$; $\left.a_{p}=0.06 \mathrm{MPa} / \mathrm{s}^{n} p ; n_{p}=0.4 ; a=15 \mathrm{~mm}\right): 1-\omega_{e c} ; 2-\omega_{e a} ; 3-\omega_{e b}$

Comparative analysis shows a good compliance of the results obtained during the experiment with the results obtained during the modeling (up to $15 \%$ ).

Conclusion. We obtained equations that allow approaching the modeling of square workpiece forming under the shortterm creep conditions. The resulting equations facilitated the theoretical evaluation of the investigated operation. We found the correspondences reflecting the influence of the loading rule, workpiece geometry and mechanical properties on stresses, deformations, operation pressure and critical modes during the forming of square workpieces under the conditions of short-term creep. The research resulted in finding the possibilities of defect-free forming of a titanium alloy obeying the kinetic theory of creep and damage. The research shows the influence of forming time flow on the material damage rate.

\section{Acknowledgment}

The work was carried out within the framework of RFBR grants No. 16-48-710016 and 16-08-00020 and Tula region administration grant.

\section{References}

[1] S.N. Larin "Pneumoforming of cellular panels from anisotropic material“, Izvestiya TulGU. Technical science, Tula: Publishing house of Tula State University, Vol. 3, pp. 51-61, 2010.

[2] S.S.Yakovlev, S.N. Larin, V.I. Tregubov, Isothermal pneumoforming of cells of cellular multi-layer sheet structures from anisotropic highstrength materials in the creep regime, Tula: Publishing House of Tula State University, 173 p., 2011.

[3] N.N. Malinin, Creep in metal processing, M .: Mechanical Engineering, 216 p., 1986.

[4] V.I. Ershov, V.I. Glazkov, M.F. Kashirin, Improvement of forming operations of sheet punching, M $\therefore$ Mechanical Engineering, 311 p., 1990

[5] Wenjun Zhao, Fuyang Cao, Xiaolong Gu, Zhiliang Ning, Ying Han, Jianfei Sun "Isothermal straining of spray formed $\mathrm{Al}-\mathrm{Zn}-\mathrm{Mg}-\mathrm{Cu}$ alloy", Mechanics of Materials, Vol. 56, pp. 95-105, 2013. 
[6] S. Rusz, J. Sinczak, W. Lapkowski "Isothermal plastic forming of highcarbon steel", Materials Science and Engineering, Vol. 234-236, 430433 pp., 1997.

[7] J.Sinczak, W. Lapkowski, S. Rusz "Isothermal plastic forming of high melting temperature alloys", Journal of Materials Processing Technology, Vol. 72, Issue 3, 429-433 pp., 1997.

[8] I. Puertas, C.J. Luis-Pérez, D. Salcedo, J. León, R. Luri, J.P. “Fuertes Isothermal Upset Forging of AA5083 after Severe Plastic Deformation by ECAE", Procedia CIRP, Vol. 12, 288-293 pp., 2013.

[9] Wan-peng Dong, Jun Chened Fea "Simulation of 4A11 piston skirt isothermal forging process", Transactions of Nonferrous Metals Society of China, Vol. 18, Issue 5, 1196-1200 pp., 2008.

[10] M. Abbasia, A. Saeed-Akbarib, M. Naderi "The effect of strain rate and deformation temperature on the characteristics of isothermally hot compressed boron-alloyed steel", Materials Science and Engineering, Vol. 538, 356-363 pp., 2012.
[11] M. Naderia, L. Durrenbergerb, A. Molinarib, W. "Blecka Constitutive relationships for $22 \mathrm{MnB} 5$ boron steel deformed isothermally at high temperatures”, Materials Science and Engineering, Vol. 478, Issues 1-2, 130-139 pp., 2008.

[12] Yu Fuxiaoa, Cui Jianzhonga, S. Ranganathanb, E.S. Dwarakadasab "Fundamental differences between spray forming and other semisolid processes", Materials Science and Engineering, Vol. 304-306, 621-626 pp., 2010.

[13] Li Lia, Xinming Zhangb "Hot compression deformation behavior and processing parameters of a cast $\mathrm{Mg}-\mathrm{Gd}-\mathrm{Y}-\mathrm{Zr}$ alloy", Materials Science and Engineering, Vol. 528, Issue 3, 1396-1401 pp., 2011.

[14] Eva-Lis Odenbergera "Thermo-mechanical material response and hot sheet metal forming of Ti-6242", Materials Science and Engineering, Vol. 489, Issues 1-2, 158-168 pp., 2008. 